(Aus dem Physiologischen Institut der Universität Kiel.)

\title{
Untersuchungen über die Wirkung der ultravioletten Strahlen auf Eiweißlösungen.
}

I. Mitteilung.

Von

Rudolf Mond,

Assistent am Institut.

Mit 8 Textabhildungen.

(Eingegangen am 5. Juli 1922).

Es ist seit langem bekannt, daß vor allem die kurzwelligen Strahlen des Spektrums die mannigfaltigsten chemischen Veränderungen zu verursachen imstande sind. Jedoch sind ihre Wirkungen auf den tierischen Organismus, die zuweilen pathologische Zustände, ja sogar den Tod herbeiführen können, in ihrem Wesen noch wenig erforscht und vielfach rätselhaft. Wohl hat man manche Veränderungen des Stoffwechsels nach Belichtung gefunden, wie Vermehrung der Sauerstoffaufnahme und der Kohlensäureausscheidung, Erhöhung des Stickstoffgehaltes des Harns bei starker Belichtung nach Injektion sensibilisierender Farbstoffe [Pincussen $\left.\left.{ }^{1}\right)\right]$, aber der Ausgangspunkt und der Verlauf der sich hierbei abspielenden chemischen Vorgänge ist nicht bekannt. Auch die Ergebnisse der Reagensglasversuche sind nicht sehr umfangreich und beschränken sich im wesentlichen auf die Feststellung einiger wichtiger Tatsachen, z. B., daß die ultravioletten Strahlen Fermente lähmen, Komplement zerstören, Eiweißkörper zum Koagulieren bringen.

Gerade das Studium der Veränderung der Eiweißlösungen durch Bestrahlung scheint einer der nächstliegenden Wege zu sein, um der Erkenntnis der Strahlenwirkung auf den tierischen Organismus näher zu kommen. Bisher liegen hierüber wenige Untersuchungen vor, deren Ergebnisse man kurz dahin zusammenfassen kann, daß Eiweißlösungen nach mehr oder weniger langer Bestrahlungszeit irreversibel geflockt werden [Chalupecky ${ }^{2}$ ), Bovie ${ }^{3}$ ), Schanz $\left.{ }^{4}\right)$ ], daß der Temperaturkoeffizient

1) Pincussen, Ergebn. d. Physiol. 19. 1921. Daselbst weitere Literatur.

2) Chalupecky, Casop. Lékaun Ceskych. 64. 1913, nach Zentralbl. f. Bioch. 18.

3) Bovie, Science 37, Nr. 970. 1913; 37, Nr. 949. 1913.

4) Schanz, Pflügers Arch. f. d. ges. Physiol. 164, 445. 1916; 1r0, 646. 1918. 
dieser Reaktion klein ist, ein Charakteristikum, das überhaupt der Lichtwirkung eigentümlich ist. Schanz hat weiterhin Stabilitätsänderungen in bezug auf die Ammonsulfatfällbarkeit vor allem der Albumine gefunden, und er schließt daraus, daß durch die Lichtwirkung Albumine in Globuline umgewandelt werden könnten, eine Annahme, die übrigens auf Grund seiner Versuche in keiner Weise gerechtfertigt erscheint.

Die Notwendigkeit, dieses bis jetzt noch wenig bekannte Gebiet systematisch zu erschließen, liegt zweif $€$ llos vor. Um mir eine gewisse Ưbersicht zu verschaffen und um gangbare Wege aufzufinden, habe ich die Veränderungen, die an den Eiweißkörpern des Plasmas durch Bestrahlung mit ultraviolettem Licht auftreten, zunächst physikalischchemisch in verschiedener Hinsicht untersucht. Die vorliegende erste Mitteilung gibt eine Zusammenstellung der bisherigen Versuchsergebnisse. Sie macht bei dem schwierigen und umfangreichen Gebiet nicht den Anspruch auf völlige Durcharbeitung. Das war bei den vielen neuen Ausblicken, die sich während der Arbeit ergaben, nicht ausführbar. Das vorliegende Material stellt zu einem Teil nur die Vorarbeiten dar, auf Grund deren es jetat möglich ist, ganz bestimmte Wege einzuschlagen.

Die Arbeit wurde von Prof. Höber zunächst mit der Absicht angeregt, den Einfluß der durch die Bestrahlung mit ultraviolettem Licht verursachten Dispersitätsänderungen von Eiweißlösungen auf die Sedimentierung der roten Blutkörperchen zu verfolgen, um damit zur Klärung der theoretischen Grundlagen dieses Vorganges beizutragen. Über die Versuchsergebnisse hierüber, die noch nicht ganz abgeschlossen sind, werde ich später berichten.

Methodik: Sämtliche Untersuchungen wurden an Pferdeblut angestellt, das je nachdem durch Einfließenlassen in 2\% Ammoniumoxalatlösung oder durch. Schlagen ungerinnbar gemacht wurde. Ich bestrahlte Plasma, Serum und die durch Fällung mit Ammonsulfat in üblicher Weise gewonnenen Fraktionen, die wieder in destilliertem Wasser gelöst und verschieden lange Zeit meistens mit $\mathbf{Z u}$ satz von Thymol, außer wenn die Oberflächenspannung gemessen wurde, dialysiert wurden. Die Zeit der Dialyse ist in jedem Versuch bezeichnet. Der Prozentgehalt an Eiweiß variiert in den einzelnen Versuchsreihen, hält sich aber im allgemeinen unterhalb der im Blut vorhandenen. Quantitative Bestimmungen führte ich nicht aus, da es in den Versuchen lediglich auf die Untersehiede zwischen bestrahlten. und unbestrahlten Proben also auf relative Werte ankommt. Als Lichtquelle diente eine Krohmeyerquarzlampe mit Wasserkühlung; die Bestrahlung wurde vorgenommen in Quarzröhrchen von $8 \mathrm{~mm}$ innerem Durchmesser, die in $10 \mathrm{~cm}$ Abstand vor der Quarzlampe aufgestellt wurden und in regelmäBigen Zeitintervallen gedreht wurden, um die Bestrahlung möglichst gleichmäßig zu gestalten. Die Wärmestrahlen waren durch die Wasserkühlung vollständig ausgeschaltet, so daB bestrahlte und unbestrahlte Proben gleiche Temperatur zeigten. Überhaupt wurde besonders darauf geachtet, daß alle Lösungen sich unter gleichen Bedingungen befanden. Mußte die Bestrahlung über mehrere Tage ausgedehnt werden, so wurden 
bestrahlte und unbestrahlte Proben nachts in den Eisschrank gestellt. Die Untersuchung des ganzen Materials fand am Schluß einer Bestrahlungsserie statt, so daß die durch das einfache Stehenlassen bewirkten Veränderungen der Eiweißlösungen alle Proben gleichmäßig betrafen.

Die Untersuchung erstreckte sich im wesentlichen auf die Bestimmung der Koagulationstemperatur, der Alkohol- und Ammonsulfatfällbarkeit, der Goldzahl, die Messung der Viscosität, der Oberflächenspannung und der $\mathrm{H}^{\prime}$-Konzentration.

Die Bestimmung der Koagulationstemperatur erfolgte so, daß je $1 \mathrm{ccm}$ der Kontrolle und der bestrahlten Lösung in gleich weiten Reagensgläsern in einem Becherglas auf dem Wasserbad allmählich unter dauerndem Umrühren erwärmt wurde. Ich unterschied zwischen Opalescenz, Trübung, fein- und grobflockiger Koagulation. Als Alkohol- bezw. Ammonsulfatzahl bezeichne ich die Menge Alkohol oder gesättigte Ammonsulfatlösung, die nötig ist, um in $1 \mathrm{ccm}$ der zu untersuchenden Lösung die erste Trübung zu erzeugen.

Die Herstellung des Goldsols erfolgte nach der von Zsigmondy angegebenen Formolmethode mit dem Unterschied, daß statt in Silberkühlern destillierten Wassers in alten Glaskiuhlern aus Jenenser Glas destilliertes Wasser verwendet wurde. Als Goldzahl bezeichne ich in meinen Versuchen nicht die Gewichtsmenge Eiweiß, sondern das Volum der zu untersuchenden Lösung, das nötig ist, um $10 \mathrm{ccm}$ Goldsol yor dem Farbumsehlag durch $3 \mathrm{ccm} 10$ proz. NaCl-Lösung zu schützen. Die Lösungen mußten immer auf das 20-50fache verdüinnt werden. Die Werte sind also auch hier lediglich relativ und nur in den einzelnen Versuchsreihen vergleichbar.

Die Viscosität wurde im Thermostaten bei $28^{\circ}$ gemessen. Als Werte sind die Ausflußgeschwindigkeiten durch ein Oswaldsches Viscosimeter eingesetzt.

Als Maß für die Oberflächenspannung gilt das Volum von drei Tropfen, bestimmt im Traubeschen Viscostagonometer bei Zimmertemperatur.

Die Messung der $\mathbf{H}^{-}$-Konzentration stellte ich teils mit der Gaskette, teils mit Indikatoren an.

Im Laufe der Untersuchungen wurde die Einführung weiterer Methoden notwendig, deren Ausführung bei den betreffenden Versuchen näher beschrieben wird.

Wenn man Pferdeplasma mit ultraviolettem Licht bestrahlt, so tritt nach wenigen Stunden eine mehr und mehr zunehmende Flockung auf. Die Farbe des dunkelgelben Plasmas blaßt allmählich ab, schon bevor sichtbare Trübungen sich zeigen, und es tritt ein eigentümlich brenzlicher Geruch auf. Bestimmt man nun die Koagulationstemperatur, so stößt man auf eigenartige Veränderungen. Fällt in der Kontrolle bei $56^{\circ}$ ein dickflockiger Niederschlag aus, so beginnt in den bestrahlten Proben bei etwa derselben Temperatur eine feine Opalescenz, die sich ganz allmählich bei weiterer Erwärmung bis auf $80^{\circ}$ zu stärkerer Trübung steigert. Zu grobflockiger Koagulation kommt es überhaupt nicht mehr. Man könnte nun zunächst annehmen, daß durch die Bestrahlung die labilsten Eiweißkörper ausgeflockt wurden und die Stabilitätserhöhung auf den übriggebliebenen Rest zurückzuführen wäre. Dem ist aber nicht so, da auch dann, wenn noch keine sichtbare Veränderung durch die Bestrahlung eingetreten ist, also sicher nichts 
ausgefallen ist, dieselben Veränderungen sich zeigen. Weiterhin ist bemerkenswert, daß dieser Zustand, der schon nach einer Bestrahlungszeit von 2-4 Stunden eintritt, bei weiterer Belichtung stationär bleibt, und die Koagulationstemperaturen sich nicht mehr nach der einen oder anderen Seite verschieben, trotzdem die Strahlungswirkung, wie Viscositäts- und Oberflächenspannungsmessungen usw., auf die ich später eingehe, beweisen, ihren stetigen Fortgang nimmt. Die folgende Versuchsreihe zeigt die Veränderungen der Viscosität, Koagulationstemperatur, Alkohol- und Ammonsulfatzahl. Das schraffierte Feld in der zugehörigen Abb. 1, sowie in den folgenden stellt die Koagulationszone dar, d. h. den Temperaturbereich vom Beginn cer Opalescenz bis zur feinflockigen Koagulation.

\section{Plasma.}

$\begin{array}{ccccc}\text { Bestr.-Dauer Std. } & \text { Koag.-Temp. } & \text { Ammonsulfat-Zahl } & \text { Alkohol-Zahl } & \text { Viscosität } \\ 0 & 55^{\circ} & 0,15 & 0,30 & 73,6 \\ 3 & 56^{\circ} & 0,15 & 0,35 & 74,8 \\ 7 & 57^{\circ} & 0,15 & 0,45 & 76,9 \\ 14 & 58^{\circ} & 0,15 & 0,45 & 77,6 \\ 26 & 61^{\circ} & 0,15 & 0,40 & 80,5\end{array}$

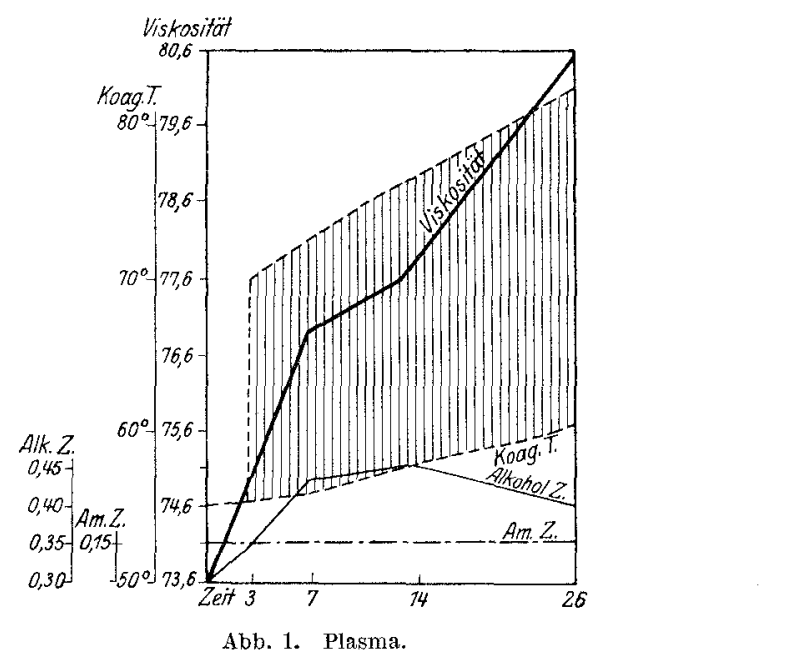

Wir finden für diese Veränderung obne weiteres keine Erklärung, denn wir müssen bedenken, daß im Plasma Eiweißkörper verschiedener isoelektrischer Punkte, Dispersitätsgrade und Quellungszustände sich befinden. Es ergibt sich daraus als nächstliegendes, die Stabilitätsänderungen der einzelnen Fraktionen zu untersuchen. 
II. Albumine.

6 Tage gegen destilliertes Wasser dialysiert.

$\begin{array}{ccccc}\text { Bestr. Dauer Std. } & \text { Koag.-Temp. } & \text { Ammonsulfat-Zahl } & \text { Alkohol-Zahl } & \text { Viscosität } \\ 0 & 70^{\circ} & 1,2 & 1,15 & 57,3 \\ 3 & 69^{\circ} & 1,1 & 1,15 & 57,6 \\ 7 & 67^{\circ} & 1,1 & 1,10 & 58,1 \\ 13 & 65^{\circ} & 1,0 & 1,00 & 58,8 \\ 21 & 63^{\circ} & 0,8 & 0,9 & 60,3 \\ 30 & 59^{\circ} & 0,75 & 0,6 & 61,3\end{array}$

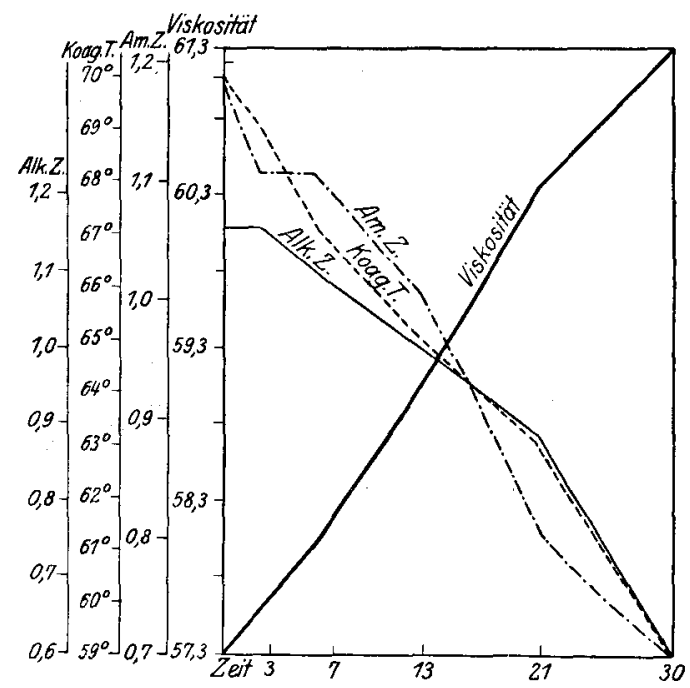

Abb. 2. Albumin.

Alkohol-, Ammonsulfatzahl nehmen stetig mit der Bestrahlungszeit $a b$, während die Viscosität ansteigt. In den drei am längsten bestrahlten Proben tritt zunehmende Trübung auf. Bei Erwärmen auf $75^{\circ}$ erstarren die 7-30 Stunden bestrahlten Lösungen zu einer festen Gallerte.

\section{Globulin + Fibrinogen.}

d. h. aus Plasma dargestellte halbgesättigte Ammonsulfatfraktion. Dialyse 3 Tage gegen distilliertes Wasser, 2 Tage gegen 0,9 proz. NaCl-Lösung.

$\begin{array}{ccccc}\text { Bestr.-Dauer Std. } & \text { Koag.-Temp. } & \text { Ammonsulfat-Zahl } & \text { Alkohol-Zahl } & \text { Viseosität } \\ 0 & 60^{\circ} & 0,25 & 0,5 & 63,6 \\ 3 & 63^{\circ} & 0,25 & 0,5 & 64,0 \\ 6 & 64^{\circ} & 0,25 & 0,6 & 64,8 \\ 9 & 65^{\circ} & 0,30 & 0,6 & 65,6 \\ 12 & 67^{\circ} & 0,30 & 0,6 & 66,0 \\ 15 & 67^{\circ} & 0,30 & 0,6 & 66,7 \\ 18 & 67^{\circ} & 0,30 & 0,6 & 67,0 \\ 21 & 67^{\circ} & 0,30 & 0,6 & 67,3 \\ 24 & 67^{\circ} & 0,30 & 0,6 & 68,4\end{array}$




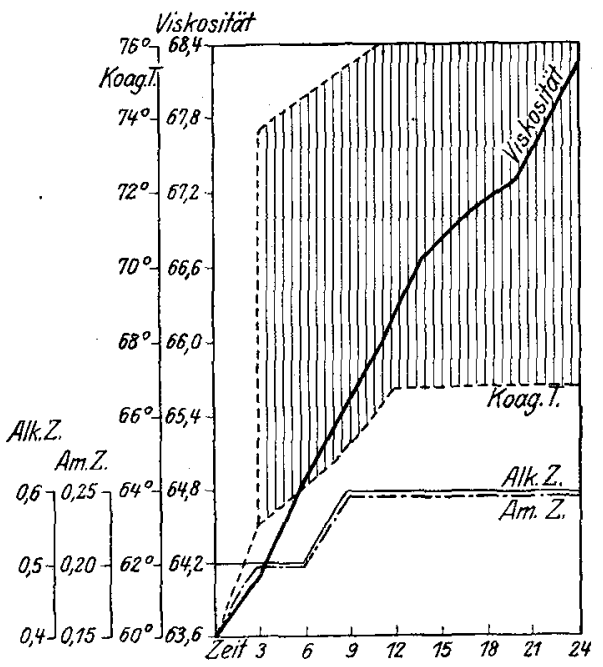

Abb. 3. Globulin + Fibrinogen.

Die Viscosität steigt auch hier und noch stärker wie bei den Albuminen an. Aber die Stabilitätsänderungen verhalten sich ganz anders und ganz ähnlich wie beim Plasma. Während Ammonsulfat- und Alkoholzahl sich nicht wesentlich verschieben, steigt die Koagulationstemperatur sehr bald an und geht in eine breite Zone feiner Trübung über.

\section{Globutin}

aus Serum durch Halbsättigung mit Ammonsulfat dargestellt, 3 Tage gegen destilliertes Wasser, 2 Tage gegen 0,9 proz. $\mathrm{NaCl}$ dialysiert.

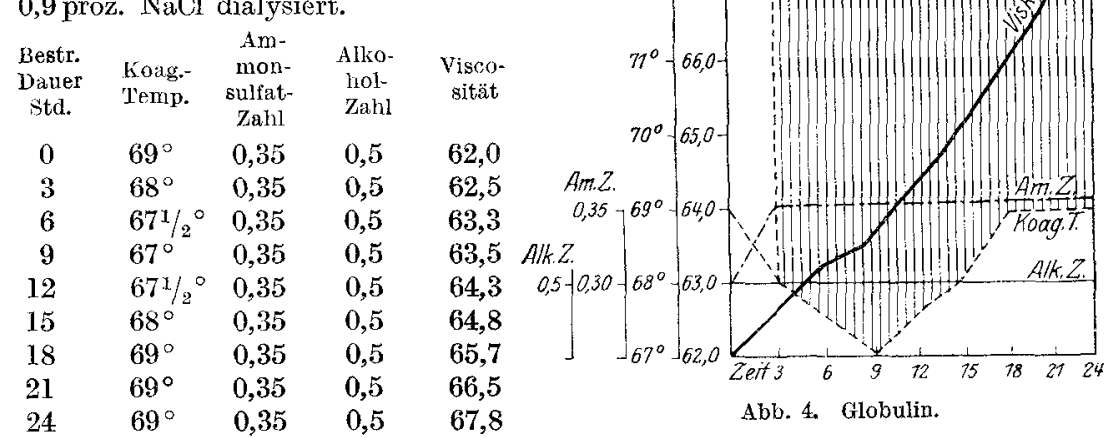

Es zeigt sich hier ein Unterschied gegen den vorigen Versuch insofern, als der Beginn der Trübung beim Erwärmen zunächst bei Bestrahlung bis zu 9 Stunden bei niederer Temperatur einsetzt, um dann allmählich wieder anzusteigen. Dieses Verhalten ist sehr bemerkenswert und ich werde später darauf zurückkommen. Offenbar geht aus 
den Kurven für das Plasma, wie aus den letzten beiden Versuchen hervor, daß die Stabilitätserhöhung gegen Erwärmung, die sich von vornherein in einem Ansteigen der Koagulationstemperatur bemerkbar macht, wahrscheinlich dem Fibrinogen zuzuschreiben ist, da sich ja das Globulin zunächst anders verhält.

Es ist mir aber in sehr vielen Versuchen nicht gelungen, diesen Unterschied zwischen dem Globulin und dem Fibrinogen quantitativ einwandfrei herauszuarbeiten. Es liegt das einmal an der Schwierigkeit, die Werte gut miteinander vergleichen zu können: Wie ich schon weiter oben anführte, äußert sich die Stabilitätserhöhung vor allem in der Art der Koagulation, in der enormen Verbreiterung der Koagulationszone und es ist da sehr schwer, für den ganz allmählichen Beginn der Opalescenz einen genauen Wert festzusetzen. Zweitens ist das Fibrinogen ein äußerst labiler Eiweißkörper, und es ist daher sehr wohl anzunehmen, daß der kolloidale Zustand einer Fibrinogenlösung von dem a priori im Plasma vorhandenen stark abweicht und daß die durch die Bestrahlung bewirkte Veränderung nicht ohne weiteres mit der im Plasma vor sich gehenden verglichen werden kann. Eine Fibrinogenlösung, die durch Ausfällen mit Ammonsulfat und Wiederauflösen des Niederschlages gewonnen wird, ist schon an sich so instabil, daß sie nach einer Bestrahlungszeit von nur wenigen Stunden schon geflockt wird.

V. Fibrinogen, dargestellt durch $3 / 10^{-S a ̈ t t i g u n g ~ d e s ~ P l a s m a s ~ m i t ~ A m m o n s u l f a t, ~}$ 3 Tage gegen $0,9 \% \mathrm{NaCl}$ dialysiert.

$\begin{array}{ccccc}\text { Bestr.-Dauer Std. } & \text { Koag.-Temp. } & \text { Ammonsulfat-Zahl } & \text { Alkohol-Zahl } & \text { Viscosität } \\ \mathbf{0} & \mathbf{5 9} & \mathbf{0 , 2 5} & \mathbf{0 , 4 5} & \mathbf{6 2 , 8} \\ \mathbf{3} & \mathbf{5 9}^{\circ} & \mathbf{0 , 2 5} & \mathbf{0 , 4 5} & \mathbf{6 3 , 7} \\ \mathbf{7} & \mathbf{5 8} & 0,25 & 0,42 & \mathbf{6 4 , 2} \\ \mathbf{1 4} & \mathbf{5 6 ^ { \circ }} & 0,25 & 0,40 & \mathbf{6 5 , 4} \\ \mathbf{2 5} & \mathbf{5 5}^{\circ} & 0,25 & 0,35 & \mathbf{6 6 , 4}\end{array}$

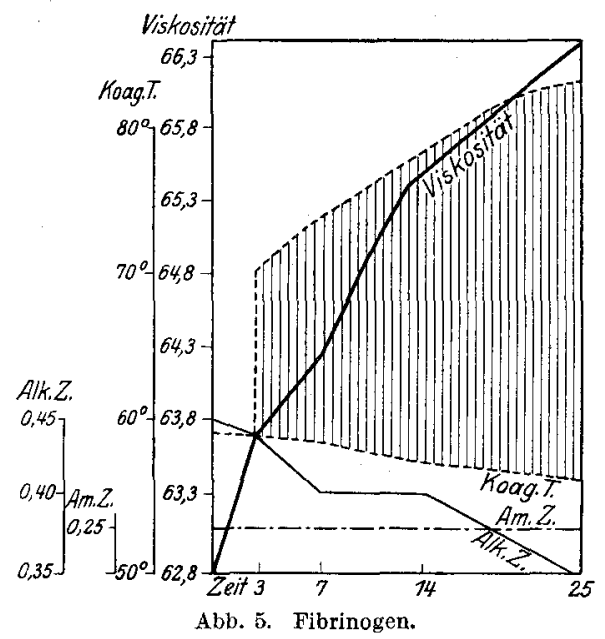


Erwähnenswert ist noch die Beobachtung, daß bis auf $80^{\circ}$ erwärmte bestrahlte Fibrinogenlösungen nicht zu einer Gallerte erstarren im Gegensatz zum .Albumin und Globulin.

In den beiden folgenden Versuchen habe ich die Globulinfraktion weiter in Eu- und Pseudoglobulin zerlegt. Man sieht, daß die Stabilitätsänderungen der Euglobulinlösung mit der der Gesamtglobulinfraktion übereinstimmen, während das Pseudoglobulin in sfinem Verhalten gegen die Bestrahlung dem Albumin näher steht.

$\begin{array}{ccccc}\text { Bestr.-Dauer Std. } & \text { Koag.-'Temp. } & \text { VI. Euglobulin. } \\ 0 & 70^{\circ} & 0,35 & 0,7 & \text { Viscositant. } \\ 3 & 70^{\circ} & 0,35 & 0,7 & 68,1 \\ 7 & 67^{\circ} & 0,35 & 0,7 & 68,6 \\ 14 & 67^{\circ} & 0,35 & 0,7 & 69,5 \\ 21 & 70^{\circ} & 0,35 & 0,7 & 70,7\end{array}$

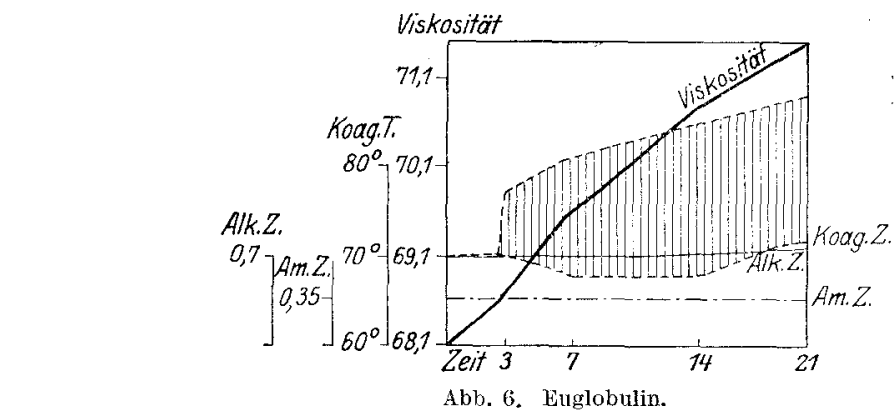

VII. Pseudoglobulin.

$\begin{array}{ccccc}\text { Bestr.-Dauer Std. } & \text { Koag.-Temp. } & \text { Ammonsulfat-Zahl } & \text { Alkohol-Zahl } & \text { Viscosität. } \\ 0 & 75^{\circ} & 1,30 & 0,7 & 51,9 \\ 3 & 75^{\circ} & 1,25 & 0,67 & 52,4 \\ 7 & 74^{\circ} & 1,15 & 0,65 & 52,7 \\ 14 & 73^{\circ} & 1,00 & 0,63 & 53,1 \\ 21 & 72^{\circ} & 0,90 & 0,6 & 53,7\end{array}$

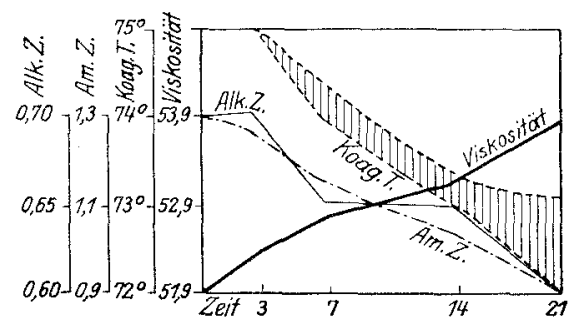

Abb. 7. Pseudoglobulin.

Ich fasse die bisher festgestellten Stabilitätsänderungen in den einzelnen Fraktionen noch einmal kurz zusammen: Allen eigentümlich 
ist eine Farbänderung der Lösungen, die während der Bestrahlung eintritt und die sich in einer Abblassung oder einem Gelberwerden des Farbtones bemerkbar macht. Weiterhin ist allen gemeinsam ein eigentümlich brenzlicher Geruch, der an Intensität mit der Bestrahlungszeit wächst. Die Albumine zeigen starke Abnahme der Koagulationstemperatur, der Alkohol- und Ammonsulfatzahl, auf $80^{\circ}$ erwärmt, gelatinieren sie, um so ausgesprochener, je länger sie bestrahlt werden. Die Globuline hingegen werden durch die Bestrahlung in ihrer Stabilität ganz anders verändert, was sich vor allem in dem eigentümlichen Verhalten der Koagulationstemperatur bemerkbar macht. Ammonsulfat und Alkoholzahl ändern sich nicht wesentlich. Auch sie gelatinieren bei Erwärmung auf $80^{\circ}$, bis auf das Fibrinogen, das aber sonst im wesentlichen dieselben Veränderungen wie das Globulin durchmacht. Die Viscosität steigt überall an, am wenigsten beim Albumin, am meisten bei der Globulintraktion.

Wir haben weiterhin Versuche darüber angestellt, ob die Funktion der Eiweißlösungen als Schutzkolloide für das kolloidale Gold durch die Bestrahlung beeinflußt wird. Es zeigten sich da nur beim Albumin geringe Unterschiede im Sinne einer Zunahme der Schutzwirkung. Die Globuline hingegen ließen keine Veränderung erkennen. Beim Fibrinogen trat bei langer Einwirkung der Strahlen eine Verminderung der Schutzwirkung auf, die aber damit erklärt werden kann, da B immer dann auch eine starke Flockung auftrat, wodurch natürlich die Konzentration der Lösung abnahm.

Folgende Versuche mögen die Veränderungen demonstrieren; die Lösungen mußten alle stark verdünnt werden.

\begin{tabular}{cc|}
\multicolumn{2}{c|}{ Albumin } \\
Bestr.-Dauer & Goldzahl \\
0 & 0,06 \\
3 & 0,06 \\
7 & 0,055 \\
14 & 0,03 \\
25 & 0,03
\end{tabular}

\begin{tabular}{lccc}
\multicolumn{4}{c}{ Albumin in Acetat-Gemischen } \\
\multicolumn{4}{c}{ Goldzahl } \\
$\mathrm{p}_{\text {H }}$ & Bestr.-Dauer & unbestr. & bestr. \\
6,0 & 6 & 0,05 & 0,04 \\
4,7 & 6 & 0,05 & 0,04 \\
3,5 & 6 & 0,03 & 0,02
\end{tabular}

\begin{tabular}{cc}
\multicolumn{2}{c}{ Globuline } \\
Bestr.-Dauer & Goldzahl \\
0 & 0,02 \\
3 & 0,02 \\
7 & 0,02 \\
14 & 0,02 \\
25 & 0,02
\end{tabular}

\begin{tabular}{cc}
\multicolumn{2}{c}{ Fibrinogen } \\
Bestr.-Dauer & Goldzahl \\
0 & 0,01 \\
3 & 0,01 \\
7 & 0,01 \\
14 & 0,015 \\
25 & 0,03
\end{tabular}

Eine Erklärung für die Ursache dieser Veränderungen können wir vorerst nicht geben, da die Theorie der Schutzwirkung noch zu wenig geklärt ist. Überdies sind mir Untersuchungen über die Funktion der Eiweißkörper als Schutzkolloide bei variierter Wasserstoffzahl nicht 
bekannt, die zeigen müßten, ob die Eiweißionen oder der undissozierte Anteil Träger der Schutzwirkung sind.

Wir haben uns bisher lediglich mit der Feststellung begnügt, daß durch die Bestrahlung von Eiweißkörpern des Pferdeplasmas Veränderungen physikalisch-chemischer Natur in verschiedener Richtung auftreten. Die weitere Fragestellung muß sich jetzt mit der Ursache dieser Prozesse und der Analyse der Strahlenwirkung befassen. Da nun, wie aus den bisherigen Versuchen zu ersehen ist, Stabilitätsänderungen eine große Rolle spielen und wiederum die Stabilität von Eiweißlösungen von der $\mathrm{H}^{*}$-Konzentration durchaus abhängig ist, so ergibt sich die zwingende Notwendigkeit, die einzelnen Eiweißkörper unter Berücksichtigung ihrer isoelektrischen Punkte bei variierter Wasserstoffzahl zu untersuchen. Eiweißkörper sind Ampholyte und bilden mit Säuren und Basen Salze, und zwar treten sie bei einer $\mathbf{H}^{*}$ Ionenkonzentration, die größer als die des isoelektrischen Punktes ist, als Kationen, bei niedrigerer $\mathrm{H}$-Ionenkonzentration als Anionen auf. Im isoelektrischen Punkte ist die Summe der Anionen und Kationenkonzentration ein Minimum, der Dissoziationsrest, d. i. das Verhältnis der undissozierten Moleküle zur Gesamtkonzentration, ein Maximum. Alkoholzahl, Viscostiät, Koagulationstemperatur haben hier ihr Minimum. Eine Albuminlösung von $p_{\mathrm{H}}=4,7$ ist isoelektrisch. Sie ist stabil und flockt im Gegensatz zum Globulin nicht, das seinen isoclektrischen Punkt bei $p_{\mathrm{H}}=5,4$ hat.

Ich habe nun zunächst untersucht, ob das für den isoelektrischen Punkt charakteristische Flockungsmaximum durch die Bestrahlung verschoben bzw. verändert wird. Zu dem Zwecke habe ich Acetatgemische verschiedener Wasserstoffzahl hergestellt, in denen nur der Gehalt an Essigsäure variierte, dagegen die Natriumacetatkonzentration konstant gehalten wurde, und die alle mit dest. Wasser auf das gleiche Volum aufgefüllt wurden. $\mathrm{Zu}$ diesen Gemischen wurde je $1 \mathrm{ccm}$ der unbestrahlten und bestrahlten Albumin- oder Globulinlösung zugefügt. Den Albuminpufferlösungen mußte dann noch, um eine Trübung zu erhalten, überall die gleiche, vorher ausprobierte Menge 96 proz. Alkohols zugegeben werden. Die Flockung wurde nach $1 / 4$ Stunde abgelesen und ist durch Kreuze bezeichnet.

$$
\begin{aligned}
& \begin{array}{cc|c|c|c|c|c|c|c|c|c}
\text { Albumine Bestr.-Dauer } & p_{\mathrm{H}} & 3,5 & 3,8 & 4,1 & 4,4 & 4,7 & 5,0 & 5,3 & 5,6 & 5,9 \\
0 & - & + & ++ & +++ & +++ & ++ & + & + & - \\
10 & & - & ++ & ++ & +++ & +++ & +++ & ++ & + & -
\end{array} \\
& \text { Globuline } \\
& \mid \begin{array}{c|c|c|c|c|c|c|c|c}
3,5 & 3,8 & 4,1 & 4,4 & 4,7 & 5,0 & 5,3 & 5,6 & 5,9 \\
+ & ++ & ++ & +++ & +++ & ++ & + & - & - \\
+++++ & +++ & +++ & +++ & +++ & ++ & + & -
\end{array}
\end{aligned}
$$


Aus den Versuchen ist ersichtlich, daß die Flockungszone nach der Bestrahlung erheblich zunimmt. Eine Verschiebung kann auf diese Weise nicht festgestellt werden. Das Flockungsmaximum für die Globuline liegt nicht, wie man erwarten sollte, bei $p_{\mathrm{H}}=5,4$, dem isoelektrischen Punkt der Globuline. Das rührt daher, daß die Globuline gegen $0,9 \%$ NaCl-Lösung dialysierten und das Cl-Anion das Flockungsmaximum nach der sauren Seite verschiebt ${ }^{1}$ ). Die Flockungszone für Fibrinogenlösungen ist zu breit, um Unterschiede erkennen zu lassen.

Weit interessanter sind die Ergebnisse der Untersuchungen, die zeigen, welche Veränderungen die Eiweißlösungen erleiden, wenn sie von vornherein bei variierter Wasserstoffzahl der Bestrahlung ausgesetzt werden.

Ich führe folgendes Versuchsbeispiel an:

VIII.

Zu je 10 com einer Albuminlösung, die 10 Tage gegen destilliertes Wasser dialysierte wurden Acetatgemische untenstehender Wasserstoffzahl zugesetzt und mit dest. Wasser auf gleiches Volum aufgefüllt.

\begin{tabular}{|c|c|c|c|c|c|c|c|}
\hline \multirow{2}{*}{$\begin{array}{c}\text { Dest. } \\
\text { Röhrch. } \\
p_{\mathbf{H}}\end{array}$} & \multirow{2}{*}{$\begin{array}{c}\text { Bestr. } \\
\text { Dauer } \\
\text { Std. }\end{array}$} & \multicolumn{2}{|c|}{ Alkoholzahl } & \multicolumn{2}{|c|}{ Ammonsulfat-ZahI } & \multicolumn{2}{|c|}{ Koag.-Temperatur } \\
\hline & & unb. & bestr. & unb. & bestr. & $\begin{array}{l}\text { unb. } \\
\text { Grad }\end{array}$ & $\begin{array}{l}\text { bestr. } \\
\text { Grad }\end{array}$ \\
\hline 16,0 & 6 & 0,9 & 0,45 & 1,25 & 1,05 & 70 & $5 \overline{9}$ \\
\hline 25,4 & 6 & 0,6 & 0,3 & 1,05 & 0,95 & 68 & 55 \\
\hline 34,7 & 6 & 0,4 & 0,2 & 0,95 & 0,70 & 61 & 52 \\
\hline $4 \quad 4,1$ & 6 & 0,35 & 0,2 & 0,70 & 0,35 & 63 & 63 \\
\hline $5 \quad 3,5$ & 6 & 0,45 & 0,4 & 0,40 & 0,25 & 80 & 80 \\
\hline
\end{tabular}

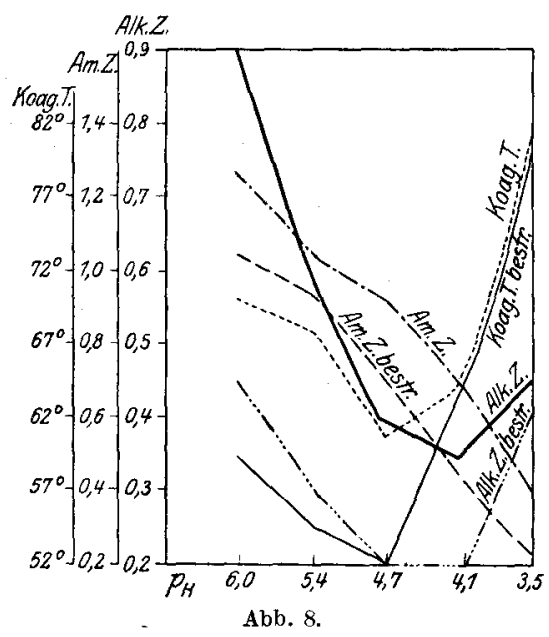

Nach 2 Stunden tritt in Röhrchen 3 leichte Opalescenz auf, die rasch zunimmt und sich gegen Ende der Bestrahlung zu starker Trübung steigert, Röhrchen 2 und 4 zeigen nach 6 Stunden geringe Opalescenz, 1 und 5 bleiben unverändert. Auf tropfenweisen Zusatz von $\mathrm{NaOH}$ tritt in 4 und 5 gleichstarke Trübung auf wie in Röhrchen 3, ein Beweis, dafür, daß Strahlenwirkung und Flokkung zwei ganz verschiedene Prozesse sind, worauf auch Bovie schon hingewiesen hat, daß also aus der Intensität der auftreten-

den Flockung keinerlei Schlußfolgerungen auf die an den Eiweißkörpern sich abspielenden Vorgänge gemacht werden können.

1) Michaelis und von Szent Györgyi, Bioch. Zeitschr. 103, 178. 1920. 
Die Betrachtung der Kurven zeigt folgendes: Alkoholzahl, Koagulationstemperatur der unbestrahlten Proben haben ihr Minimum im isoelektrischen Punkt, weil die Albuminlösung hier am instabilsten ist, der Dissoziationsrest ein Maximum hat. Je mehr Eiweiß ionisiert, um so mehr steigt die Koagulationstemperatur und die Alkoholzahl. Die Ammonsulfatzahl hingegen ist lediglich abhängig von der $\mathrm{H}^{*}$-Konzentration, ganz unabhängig vom isoelektrischen Punkt und nimmt mit fallendem $p_{\mathbf{H}}$ proportional ab. Wenn wir jetzt den Verlauf der Veränderungen in den bestrahlten Proben verfolgen, so zeigen sich merkwürdige Differenzen. Die Ammonsulfatzahl nimmt überall gleichmäßig ab und ihre Kurve läuft innerhalb der Versuchsfehler der der Kontrolle parallel, das heißt also, die Bestrahlung hat überall zu der gleichen Verminderung der Stabilität geführt. Ganz anders verlaufen die Kurven für die Alkoholzahl und die Koagulationstemperatur. Auf der alkalischen Seite des isoelektrischen Punktes sind die Differenzen zwischen Kontrolle and bestrahltem Albumin sehr erheblich, nehmen immer mehr und mehr ab und verschwinden auf der sauren Seite vom isoelektrisehen Punkt fast vollkommen. Ich sagte vorher, daß auf Zusatz von NaOH in Röhrchen 4 und 5 dieselbe Trübung auftrat wie in 3, daß also, wie auch die Ammonsulfatkurve bewcist, die absolute Stabilitätsverminderung überall dieselbe ist. Die Flockung durch Hitze oder Alkohol aber ist abhängig vom Ionisationszustand des EiweiB, und dieser von der $\mathrm{H}^{*}$-Konzentration der Lösung. Um uns nun den Verlauf der Kurven der bestrahlten Proben auf der alkalischen Seite vom isoelektrischen Punkt zu erklären, könnten wir die Annahme machen, daß die Lösungen durch die Strahlenwirkung etwas saurer geworden wären, und damit die Konzentration der undissozierten Moleküle zugenommen hätte. Mit dieser Erklärung würde auch die Beobachtung in Einklang zu bringen sein, die sich aus der Betrachtung der Kurven ergibt, daß nämlich das Stabilitütsminimum für Alkohol- und Ammonsulfatfällbarkeit in den bestrahlten Lösungen etwas nach links verschoben ist.

Ich stellte nun zunächst orientierende Versuche mit der Indicatorenmethode an, die in Albumin- und Globulinlösungen einen Anstieg der $H^{*}$-Konzentration nach 6-10 stündiger Bestrahlung bis fast um eine Zehnerpotenz ergaben. Um genauere Werte zu erhalten und um den Eiweiß- und Salzfehler der Indicatoren auszuschalten, habe ich dann eine größere Zahl von Messungen in der Wasserstoffkette angestellt und diese Werte mit denen der Indicatorenmethode verglichen. Es stellten sich bald erhebliche Schwierigkeiten ein, die ich näher darlegen werde. Ich benutzte anfangs Elektroden mit strömendem Wasserstoff und fand da immer $p_{\mathrm{m}}$-Differenzen, die erheblich niedriger waren, wie die mit Indikatoren erhaltenen. Wir nahmen dann an, daß vielleicht 
Kohlensäure sich bildete, die durch die Wasserstoffdurchleitung ausgetrieben würde. Wenn nun durch eine bestrahlte Globulinlösung, der Methylrot als Indikator zugesetzt war, Wasserstoff längere Zeit geleitet wurde, so wurde die Lösung deutlich gelber, aber wurde nie so gelb wie die unbestrahlte Kontrolle, auch nicht bei noch so langer Durchleitung von Wasserstoff. Um das Entweichen der anscheinend sich bildenden Kohlensäure zu verhüten, habe ich Elektroden mit stehender Gasblase verwendet. Auch hier zeigten sich bei den Messungen wieder Utberraschungen. Es stellte sich nämlich heraus, daß Serum, Globuline, Albumine gewonnen aus dem Blut verschiedener Tiere, sich ganz different verhielten. Zwar konnte ich fast immer eine Säuerung feststellen, außer in ganz seltenen Fällen, wo erst nach sehr langer Bestrahlungszeit eine geringe Konzentrationszunahme der H-Ionen auftrat; jedoch war der Grad der $p_{\mathrm{H}}$-Differenz zwischen bestrahlten und unbestrahlten Lösungen ein ganz verschiedener.

Zur Erläuterung führe ich folgende Versuchsbeispiele an:

$\begin{array}{cccc}\text { Serum : Bestr.-Dauer } & p_{\mathrm{H}} & \text { Serum : Bestr.-Dauer } & p_{\mathrm{H}} \\ 0 & 7,44 & 0 & 7,52 \\ 1 & 7.31 & 2^{1} / 2 & 7,45 \\ 2 & 7,30 & 4 & 7,40 \\ 3 & 7,18 & 5 & 7,41 \\ 5 & 7,14 & 7 & 7,40 \\ 6 & 7,07 & 9 & 7,25 \\ 7 & 6,92 & & \\ 8 & 6,87 & & \\ \text { Globuline : Bestr.-Dauer } & p_{\mathrm{H}} & \text { Globuline : Bestr.-Dauer } & p_{\mathrm{H}} \\ 0 & 5,54 & 0 & 5,85 \\ 3 & 5,36 & 3 & 5,78 \\ 6 & 5,05 & 6 & 5,71 \\ 8 & 4,84 & 9 & 5,66 \\ \text { Albumine : Bestr.-Dauer } & p_{\mathrm{H}} & \text { Albumine : Bestr.-Dauer } & p_{\mathrm{H}} \\ 0 & 6,12 & 0 & 6,04 \\ 3 & \mathbf{6 , 1 2} & 21 / 2 & 6,01 \\ 6 & 6,05 & 5 & 5,94 \\ 8 & 6,06 & 8 & 5,90 \\ & & 10 & 5,85\end{array}$

Es ist durchaus nötig, daß diese wichtigen und auffälligen Veränderungen weiter aufgeklärt werden. Die großen Differenzen, die in der $\mathrm{H}^{*}$-Verschiebung bei Bestrahlung verschiedener Sera auftreten und die uns vorläufig ganz unverständlich sind, lassen den Gedanken aufkommen, daß möglicherweise in manchen Sera Stoffe vorhanden sind, die besonders empfindlich auf die Bestrahlung reagieren. Natürlich können diese Substanzen auch an die verschiedenen Eiweißfraktionen adsorbiert sein, da ja die mittels fraktionierter Fällung mit Ammonsulfat gewonnenen Eiweißkörper keineswegs als rein bezeichnet werden 
können. Sollte dieser Fall zutreffen, so würden dadurch der weiteren Untersuchung und der chemischen Analyse erhebliche Schwierigkeiten in den Weg gelegt, andrerseits bleibt aber die biologische Wichtigkeit der Tatsache ungeschmälert. Schon allein die Möglichkeit einer immerhin für den Organismus beträchtlichen Säurebildung kann uns zum Teil die Entzündungserscheinungen erklären, die bei gewissen Krankheiten durch Lichtwirkung hervorgerufen werden. Man könnte da allerdings einwenden, im Körper würde die Säure so schnell neutralisiert, daß es durch Säuerung allein nicht zu Entzündungserscheinungen kommen könnte. Nun werden aber die ultravioletten Strahlen schon zum allergrößten Teil in der Epidermis absorbiert, ihre Wirkung kommt also vor allem in den oberflächlichen Schichten zum Ausdruck, und hier kann sich sehr wohl eine lokale Säurebildung vollziehen, ohne daß sie schnell neutralisiert zu werden braucht. Wie dem auch sei, jedenfalls ist für uns die durch die Bestrahlung bewirkte Verschiebung der $\mathrm{H}^{*}$ ein Indikator dafür, daß recht bedeutungsvolle chemische Umwandlungen vor sich gehen müssen. Wie ich schon weiter oben sagte, vermuteten wir, daß auch Kohlensäure abgespalten würde. Wenn sich diese Annahme bestätigt und wenn die Kohlensäure dem Eiweiß entstammt, dann müssen ganz außerordentlich starke Abbauprozesse vor sich gehen. Eine weitere Ursache für die Säuerung wäre in einer Umlagerung in der Peptidbindung, einem Übergang von der Laktim- in die Laktamform zu suchen,

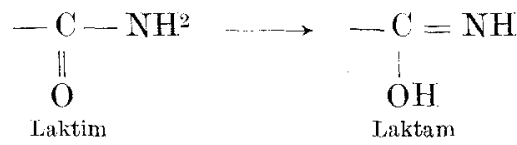

wie sie von Fernau und Pauli ${ }^{1}$ ) bei der Verschiebung der $\mathrm{H}^{-}$-Konzentration, die sie bei wochenlanger Radiumbestrahlung von Albuminlösungen beobachteten, angenommen wurde.

Es schien mir wegen der Unübersichtlichkeit der Verhältnisse durchaus notwendig, die Versuche, wie sie in der bisherigen Weise ausgeführt wurden, abzubrechen und sie an reinen Eiweißkörpern, wie z. B. dem krystallisierten Albumin, fortzuführen. Diese Untersuchungen sind noch in den Anfängen und ich werde darüber in einer späteren Mitteilung berichten.

Ich habe anfangs geglaubt, die Stabilitätserhöhungen, die sich im Globulin und Fibrinogen, vor allem im Verhalten der Koagulationstemperatur zeigen, auf die Verschiebung der $\mathrm{H}^{\prime}$-Konzentration zurückführen zu können, indem ich annahm, daß diese Eiweißkörper, deren isoelektrischer Punkt dem Nentralpunkt bedeutend näher liegt wie der des Albumins, durch die Bestrahlungswirkung über ihren iso-

1) Fernau und Pauli, Koll. Ztschrft. Bd. XXX, Heft 1, 1922. 
elektrischen Punkt hinaus in Kationen umgewandelt, die Albumine hingegen, die eine stetige Stabilitätsverminderung zeigen, diesem zugeführt würden. Diese Auffassung ist aber offenbar unhaltbar geworden. Wenn ich auch bei den Globulinen öfters zunächst einen Abfall der Koagulationstemperatur wahrnahm, die dann später wieder anstieg, so zeigte sich doch schon in der allerersten Zeit der Bestrahlung eine außerordentliche Verbreiterung der Koagulationszone, und es kam überhaupt nicht mehr zu grober Flockung bei Erwärmung bis zu $80^{\circ}$. Es müßten ja die Globuline, wenn sie ihren isoelektrischen Punkt durch die Einwirkung der Strahlung erreicht hätten, ausflocken und dann erst könnte eine Peptisation eintreten. Nun waren meine Globulinlösungen stets ammonsulfat- und kochsalzhaltig. Das Flockungsmaximum wird dureh die Anionwirkung nach der sauren Seite verschoben. Wir hätten insofern praktisch dieselben Verhältnisse wie beim Albumin. Wir müßten also, wenn die $\mathrm{H}^{*}$-Konzentration allein ausschlaggebend wäre, einen ähnlichen Verlauf der Koagulationstemperaturkurve erwarten wie beim Albumin. Ausschlaggebend ist ferner, daß die Stabilitätserhöhung immer eintritt, während die Verschiebung der $\mathrm{H}^{-}$-Konzentration von ganz wechselnder Intensität ist. Es handelt sich bei dem entgegengesetzten Verhalten der Albumine und Globuline wohl um Dispersitätsänderungen, die vielleicht durch eine Umwandlung in der chemisehen Struktur bedingt sind. Wir dürfen auch nicht vergessen, daß wir bei Betrachtung der Vorgänge nicht nur die Ampholytnatur der Eiweißlösungen zu berücksichtigen haben, sondern auch die Veränderungen, die sich an den undissoziierten Molekülen und Molekülaggregaten, die zweifellos durch die Strahlenwirkung suspensoiden Charakter erhalten, nicht vernachlässigen dürfen. Welche Wirkung hier die Bestrahlung hat, ob vielleicht der Hallwachseffekt in Betracht kommt, vermag ich jetzt noch nicht zu entscheiden.

\section{Oberflächenspannung.}

Die Änderung der Oberflächenspannung durch die Bestrahlung ist sehr ausgesprochen, und zwar nimmt das Tropfenvolum von Globulinlösungen und Serum erheblich, das von Albuminlösungen weniger stark ab.

$\begin{array}{cccc}\text { Globuline : Bestr.-Dauer } & \text { Tropfenvolum } & \text { Serum : Bestr.-Dauer } & \text { Tropfenvolum } \\ 0 & 20,1 & 0 & 23,4 \\ 4 & 19,5 & 4 & 23,0 \\ 8 & 18,9 & 8 & 22,6 \\ 11 & 18,2 & 11 & 22,1 \\ & \text { Albumin : Bestr.-Dauer } & \text { Tropfenvolum } & \\ & 0 & 22,3 & \\ 4 & 22,2 & \\ 8 & 22,0 & \\ 11 & 21,8 & \end{array}$


Der Messung der Oberflächenspannung von Fibrinogenlösungen stellten sich große Schwierigkeiten entgegen. Die Lösungen waren immer stark fadenziehend, sehr klebrig, auch bei starker Verdünnung, so daß weder mit der Tropfenmethode noch der Bestimmung der capillaren Steighöhe brauchbare Werte zu erzielen waren. Auch bei Anwendung der Methode, die Czapek beschrieben hat, und bei der man den Druck mißt, der nötig ist, um eine Luftblase zum Abreißen zu bringen, erhielt ich Werte, die nicht reproduzierbar sind. Es ist ja auch begreiflich, daß eine klebrige Flüssigkeit, die dem Gelzustand nahe steht, auch der Bildung einer Luftblase einen erheblichen mechanischen Widerstand entgegensetzt. Beim Pferdeplasma liegen die Verhältnisse ähnlich.

Wir müssen uns nun fragen, wovon die Oberflächenspannungsänderung einer Eiweißlösung abhängig sein kann. Zunächst interessiert uns das Verhalten bei variierter Wasserstoffzahl. Darüber sind Untersuchungen von Bottazzi $i^{1}$ ) und seinen Schülern angestellt worden, die besagen, daß die Oberflächenspannungserniedrigung mit der Zunahme des Dissoziationsrestes wächst, da $B$ also im isoelektrischen Punkt ein Ninimum der Oberflächenspannung liegt. Das stimmt auch überein mit dem Verhalten vieler oberflächenaktiver Körper, die undissoziiert viel capillaraktiver sind wie ihre stark dissoziierten Salze. Weiterhin ist die Möglichkeit gegeben, daß Dispersitätsänderungen die Oberflächenspannung beeinflussen, und endlich können durch die Bestrahlung capillaraktive Stoffe abgespalten werden.

Folgender Versuch möge den Verlauf der Änderungen demonstrieren:

Pferdeserum wurde 5 Tage gegen dest. Wasser dialysiert, die ausgefallenen Globuline in der Zentrifuge mit Wasser gewaschen, aufgeschwemmt, durch tropfenweisen Zusatz von $\mathrm{NaOH}$ gelöst, mit $\mathrm{n} /{ }_{10} \mathrm{HCl}$ bis zur stärksten Trübung versetzt. Die Suspension wurde in 4 Reagensgläser verteilt. $\mathrm{Zu} 1 \mathrm{n} / 10 \mathrm{NaOH}$, zu 3 und 4 $\mathrm{n} / 10 \mathrm{HCl}$ zugetropft und mit Wasser auf gleiches Volum aufgefüllt. Die H-Konzentrationen wurden in der Gaskette gemessen.

\begin{tabular}{cccccc} 
& & \multicolumn{4}{c}{ Tropfenvolum } \\
1 & & Bestr.-Dauer Std. & unbestr. & bestr. & Differenz \\
2 & $p_{\mathrm{H}}=8,02$ & 9 & 25,6 & 23,4 & 2,2 \\
3 & $p_{\mathrm{HE}}=5,76$ & 9 & 23,1 & 22,7 & 2,4 \\
4 & $p_{\mathrm{H}}=3,03$ & 9 & 25,7 & 24,2 & 1,5 \\
& $p_{\mathrm{H}}=2,31$ & 9 & 25,9 & 25,0 & 0,9
\end{tabular}

Es ist aus dem Versuch ersichtlich, daß in der Gegend des isoelektrischen Punktes ein Minimum der Oberflächenspannung liegt, sowohl bei bestrahlten, wie unbestrahlten Proben. Die Differenz des Tropfenvolumens zwischen bestrahlter und unbestrahlter Lösung auf der alkalischen Seite des isoelektrischen Punktes ist erheblich größer als auf der sauren. Die Deutung ist nicht ohne weiteres klar. Es besteht

1) Bottazzi und Agostino, Rendic. D. R. Acc. Dei Lincei 21, 561. 1912. 
neben Dispersitätsverschiedenheiten die Möglichkeit einer differenten chemischen Veränderung. Da nämlich die Globuline, wie es die kürzlich erschienenen interessanten Untersuchungen von Jarisch ${ }^{1}$ ) wahrscheinlich machen, Adsorptionsverbindungen mit Fettsäuren oder Lipoiden sind, so ist es leicht denkbar, daß auf der alkalischen Seite vom isoelektrischen Punkt diese Bindungen durch die Bestrahlung leichter gelöst werden als auf der sauren. Die Verhältnisse müssen durch weitere Versuche geklärt werden.

\section{Viscosität.}

Ich komme jetzt auf die Änderung der inneren Reibung zu sprechen, die bei der Bestrahlung von Eiweißlösungen zutage tritt, und die sich überall in sehr ausgesprochener Weise in einer beträchtlichen Erhöhung der Viscosität zu erkennen gibt. Am meisten betroffen werden die instabilen Fraktionen, das Globulin und Fibrinogen, am wenigsten das Albumin. Zweifellos ist es ein und derselbe Prozeß, der sich hier abspielt. Um aber aus den Viscositätsbestimmungen auf die Strahlenwirkung Schlüsse ziehen zu können, müssen wir uns über die Ursache der inneren Reibung von Eiweißlösungen Klarheit verschaffen. Es ist von Laqueur und Sackur zuerst die Viscosität von Eiweißlösungen bei variierter Wasserstoffzahl gemessen worden, und diese Autoren haben gefunden, daß die innere Reibung im isoelektrischen Punkt ihr Minimum hat, zu beiden Seiten ansteigt, ein Maximum erreicht und dann wieder abfällt. Laqueur und Sackur haben angenommen, daß die Eiweißionen die Träger der inneren Reibung sind, und $P a u l i{ }^{2}$ ), der sehr eingehende Untersuchungen über diese Frage anstellte, schließt sich dieser Meinung an, indem er annimmt, daß die Tonen deshalb für die Erhöhung der inneren Reibung verantwortlich zu machen sind, weil sie hydratisiert sind. Seine Untersuchungen sowie die seiner Mitarbeiter stimmen mit dieser Annahme sehr gut überein. In den Versuchen, die mit wochenlang dialysiertem Albumin angestellt sind, wird gezeigt, daß das Maximum der Viscosität mit dem Maximum der Ionisation zusammenfällt. Wenn wir aber nun diese Pauli sche Hydratationstheorie auf unsere Versuchsergebnisse anwenden wollen, so wird es uns ganz unmöglich, eine Erklärung für die Viscositätssteigerungen zu geben, die durch die Bestrahlung entstehen. Es ist ganz offensichtlich, daß hier die Viscositätszunahme ganz unabhängig davon auftritt, welche $H^{-}$-Konzentration gewählt wird, im Gegenteil gerade im isoelektrischen Punkt, wo also eine Hydratation nicht in Betracht kommt, findet eine enorme Viscositätssteigerung statt.

Als Beispiel führe ich folgenden Versuch an:

1) Jarisch, Pflügers Arch. f. d. ges. Physiol. 194, 337. 1922.

2) Pauli, Kolloidchemie der Eiweißkörper, 1. Hälfte. Dresden u. Leipzig 1920. 
Albumin in Acetatgemischen.

\begin{tabular}{lcccc} 
& \multicolumn{3}{c}{ Viscosität } & \\
$p_{\mathrm{H}}=\mathbf{5}$ & Bestr.-Dauer Std. & unbestr. & bestr. & Differenz \\
$p_{\mathrm{H}}=5,4$ & 6 & 49,0 & 49,3 & 0,3 \\
$p_{\mathrm{H}}=4,7$ & 6 & 48,8 & 49,3 & 0,5 \\
$p_{\mathrm{H}}=\mathbf{4 , 1}$ & 6 & 48,7 & 52,0 & 3,3 \\
$p_{\mathrm{H}}=3,5$ & 6 & 49,5 & 51,2 & 1,7 \\
& 6 & 51,7 & 52,1 & 0,4
\end{tabular}

Aus der Differenz der Viscosität zwischen bestrahltem und unbestrahltem Albumin sollte man schließen, daß nicht die Eiweißionen, sondern gerade der undissoziierte Anteil, und zwar dessen Fähigkeit, Aggregate zu bilden, worunter ich nicht nur sichtbare Komplexe verstehe, sondern gerade Zusammenlagerungen der Moleküle zu submikroskopischen Massen, die die Lösung nicht trüben, die Ursache der Viscositätssteigerung sind.

Diese Annahme findet in den kürzlich veröffentlichten sehr eingehenden Untersuchungen von Jaques $L_{0} b^{1}$ ) eine außerordentliche Stütze. Loeb fand, daß Aminosäuren und krystallisiertes Eieralbumin bei variierter Wasserstoffzahl in sehr weiten Grenzen überall gleiche Viscosität zeigten, daß erst bei einem sehr niedrigen $p_{\mathrm{H}}$, etwa bei 1,0 , die Albuminlösung einen Anstieg in der inneren Reibung hatte. Er schließt daraus, daß die Eiweißionen nicht die Träger der inneren Reibung sein können. In seiner theoretischen Betrachtung geht er aus von der Formel, die von Linstein gefunden wurde,

$$
\eta=\eta_{0}(1+2,5 \varphi)
$$

worin $\eta$ die innere Reibung der Lösung, $\eta_{0}$ die des Lösungsmittels und $\varphi$ das Verhältnis des Volums der dispersen Phase zum Gesamtvolumen und 2,5 eine Konstante bedeutet. Die Formel gilt nur dann, wenn $\varphi$ klein ist und der Radius der suspendierten Partikel klein ist im Verhältnis zu ihrem Abstand voneinander. Diese Formel hat dann später weitere Umformungen erhalten, vor allem von Arrhenius, der sie in eine auch für Systeme, in denen $\varphi$ verhältnismäßig groß ist, anwendbare Form gebracht hat:

$$
\log \eta-\log \eta_{0}=\delta \varphi .
$$

Loeb weist nun experimentell für Albuminlösungen, Gelatinesuspensionen und -Lösungen sowie für Caseinlösungen nach, daß die gemessene Viscosität mit der aus dem Volumen der dispersen Phase errechneten übereinstimmt. Er weist ferner nach, daß das Volumen lediglich abhängig ist von den Neutralteilchen bzw. den Aggregaten, von derem Gehalt an eingeschlossenem Wasser wiederum die Volumen-

1) J. Loeb, Journ. of Gen. Physiol. 3, 827. 1920-21; 4, 73, 97. 192I-22. Siehe ferner J. Loeb, Proteins and the Theory of Colloidal Behavior, New York 1922. 
änderung abhängt. Er findet genau wie Pauli im isoelektrischen Punkt ein Minimum der Viscosität, des osmotischen Druckes, der Leitfähigkeit und sowohl auf der alkalischen wie auf der sauren. Seite einen Anstieg. Loeb führt weiter aus, daß der Wassergehalt der Aggregate, auf den es ankommt, reguliert wird durch das Donnan gleichgewicht, das sich immer dann einstellt, wenn ein nicht permeables Ion in einer durch eine Membran von Wasser getrennten Lösung vorhanden ist. Als Membran fungiert das durch Aneinanderlagerung der Micellen gebildete Aggregat, das nicht permeable Ion ist das betreffende Proteinanion oder Kation, je nachdem $p_{\mathrm{H}}$ größer oder kleiner ist wie $p_{\mathrm{H}}$ des isoelektrischen Punktes. Daß tatsächlich ein Donnangleichgewicht sich ausbildet, beweisen die Potentialdifferenzen, die zwischen Suspensionsmittel und disperser Phase auftreten und die sowohl berechnet wie gemessen wurden, und daß jenes den Verlauf der Viscositätskurve bestimmt, zeigt ein sehr eindeutiger Versuch. Loeb suspendierte Caseinchlorid verschiedener $\mathrm{H}^{*}$-Konzentration, wartete 1 Stunde bis zur Ausbildung des Donnan gleichgewichtes und fand dann bei der $\mathbf{H}^{*}$ Konzentration des Schwellungsmaximums einen erheblichen Anstieg der Viscosität. Nach weiteren 21 Stunden war das Caseinchlorid teilweise in Lösung gegang $\mathbf{n}$ und ionisiert, die Viscositätsmessung ergab dann beim $p_{\mathrm{H}}$ des Maximums der Ionisation einen deutlichen Abfall der inneren Reibung.

Für unsere Betrachtungen haben wir in Ubereinstimmung mit den Untersuchungen Loebs daran festzuhalten, daß die Steigerung der Viscosität, die durch die Bestrahlung von Eiweißlösungen durch ultraviolette Strahlen bewirkt wird, nur auf Aggregatbildung beruhen kann, das Wachsen des Quotienten $\varphi=\frac{\text { Volum der disperen Phase }}{\text { Gesamtrolum }}$ wird bedingt durch das Wasser, welches bei der Zusammenlagerung der Micellen zu Aggregaten eingeschlossen wird. Die Viscositätsmessungen geben also sowohl einen wichtigen Einblick in die Theorie der inneren Reibung von Eiweißlösungen, als auch sind sie ein feiner Indicator für einen Teil der Veränderungen, die durch die Bestrahlung auftreten. Es ist ja schon länger bekannt, daß man durch ultraviolettes Licht Eiweißlösungen ausflocken kann, aber durch die Viscositätssteigerung wird schon der erste Beginn der Aggregatbildung angezeigt, der dann schließlich zur Koagulation führt.

In diesem Zusammenhange möchte ich noch auf einen Zustand hinweisen, der oft mit der inneren Reibung verwechselt wird; das ist die Klebrigkeit. Ich habe schon weiter oben bei Besprechung der Oberflächenspannungsänderung darauf hingewiesen, daß Fibrinogenlösungen, die eine viel schnellere Ausflußgeschwindigkeit durch ein Viscosimeter haben, wie z. B. Serum stark fadenziehend und klebrig 
sind, so daß es nicht möglich ist, die Capillaraktivität sqlcher Lösungen einwandfrei zu bestimmen. Es handelt sich hier zweifellos um einen Zustand, der der Gelbildung nahe steht, vielleicht bedingt durch eine netzförmige Aneinanderlagerung der Micellen. Daß es sich hier nicht nur um eine Zustandsänderung handelt, wie sie durch das Fällen und Wiederlösen des Niederschlages bedingt sein kann, geht daraus hervor, daß ich öfters frisches Pferdeplasma untersucht habe, daß ausgesprochen fadenziehend war. Die Theorie dieser schwierigen Verhältnisse ist von Hess und Hatschek dargelegt worden ${ }^{\mathbf{1}}$ ).

\section{Zusammenfassung.}

Mit ultraviolettem Licht bestrahlte Globuline und Fibrinogenlösungen zeigen Stabilitätserhöhungen, die sich in einem Anstieg der Koagulationstemperatur und einer Verbreiterung der Koagulationszone bemerkbar machen. Ammonsulfat- und Alkoholzahl bleiben unverändert oder lassen nur geringe Differenzen erkennen. Dagegen nehmen Koagulationstemperatur, Ammonsulfat- und Alkoholzahl der Albumine proportional mit der Bestrahlungsdauer ab.

Die Goldzahl bestrahlter' Globuline und des Fibrinogens ändert sich nicht, während beim Albumin geringe Erhöhung der Schutzwirkung eintritt.

Durch die Bestrahlung von Serum, Albumin und Globulin wird eine Verschiebung der $\mathrm{H}^{-}$-Konzentration nach der sauren Seite bewirkt, die aber nicht immer konstant ist und deren Intensität anscheinend bei verschiedenen Sera verschieden stark ist.

Die Oberflächenspannung nimmt proportional der Bestrahlungszeit $a b$, am wenigsten beim Albumin. Thre Veränderung ist abhängig von der $\mathrm{H}^{*}$-Konzentration.

Die innere Reibung nimmt überall mit der Bestrahlungsdauer zu. Es wird gezeigt, daß sie von der Aggregatbildung abhängig ist, was im Einklang steht mit der kürzlich von $J$. Loeb entwickelten Theorie der Viscosität von Eiweißlösungen.

I) Zitiert nach Freundlich, Capillarchemie. Leipzig 1922. 